\title{
Deep View-Aware Metric Learning for Person Re-Identification
}

\author{
Pu Chen, Xinyi Xu and Cheng Deng* \\ School of Electronic Engineering, Xidian University, Xi' an 710071, China \\ puchen@ @stu.xidian.edu.cn, xyxu.xd@gmail.com, chdeng@mail.xidian.edu.cn
}

\begin{abstract}
Person re-identification remains a challenging issue due to the dramatic changes in visual appearance caused by the variations in camera views, human pose, and background clutter. In this paper, we propose a deep view-aware metric learning (DVAML) model, where image pairs with similar and dissimilar views are projected into different feature subspaces, which can discover the intrinsic relevance between image pairs from different aspects. Additionally, we employ multiple metrics to jointly learn feature subspaces on which the relevance between image pairs are explicitly captured and thus greatly promoting the retrieval accuracy. Extensive experiment results on datasets CUHK01, CUHK03, and PRID2011 demonstrate the superiority of our method compared with state-of-the-art approaches.
\end{abstract}

\section{Introduction}

Person re-identification attracts increasing attentions due to its importance in many applications, e.g., video surveillance, pedestrian retrieval, and human-computer interaction. Although significant progress has been made in these years, there are still some challenging problems existing in person re-identification: 1) dramatic changes in visual appearance; 2) dissimilarity between two images of the same pedestrian; 3) similarity between two images of different pedestrians.

To address these issues, typical methods usually focus on extracting robust features or finding a similarity measure. For instance, semantic features from different body regions are captured through a multi-stage ROI pooling pipeline [Zhao et al., 2017]. In [Zhang et al., 2016], a semantics-aware image representation is learned to capture the intrinsic structure information of persons. In [Cheng et al., 2016], an enhanced triplet loss function is proposed to learn a distance measure between two pedestrian images.

However, these approaches are incapable to efficiently discover the intrinsic relevance between image pairs. Fortunately, we find that the intrinsic relevance between image pairs in similar views is different with that in dissimilar views. As shown in Figure 1(a) and (b), the image pairs of the same

\footnotetext{
${ }^{*}$ Corresponding author
}

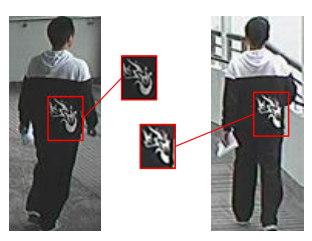

(a)

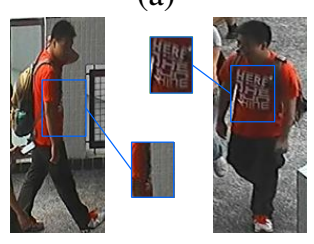

(c)

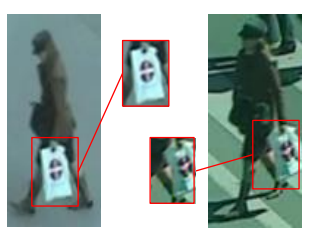

(b)

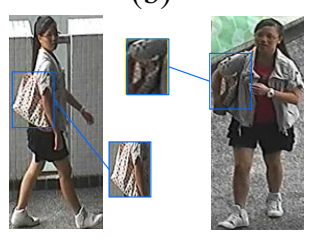

(d)
Figure 1: Examples in datasets CUHK03, CUHK01, and PRID2011. (a) and (b) show the image pairs of the same pedestrian in similar views. (c) and (d) show the image pairs in dissimilar views.

pedestrian captured from similar views can be connected by some details, such as the patterns of clothing or the carried stuff. As shown in Figure 1(c) and (d), the appearances of pedestrians change dramatically and some details are missed when the image pairs of the same pedestrian are captured from dissimilar views. Even so, the image pairs can also be connected by some view-robust features. Therefore, we can exploit the views as the supplementary information to capture the intrinsic relevance between image pairs.

In this paper, we propose a novel approach called deep view-aware metric learning (DVAML) model, where the image pairs are projected into different feature subspaces according to their view information, which can discover the intrinsic relevance between image pairs from different aspects. Multiple metrics are exploited to supervise the learning of the different feature subspaces. Extensive experiments conducted on three datasets including CUHK01, CUHK03, and PRID2011 show the effectiveness of our method compared with state-of-the-art approaches.

\section{Related Work}

Typical person re-identification methods usually focus on feature extraction or metric learning. Widely-used features include histograms [Li and Wang, 2013; Khamis et al., 2014; 
Zhao et al., 2013a], local binary patterns (LBP) [Li and Wang, 2013; Zhao et al., 2013a; Khamis et al., 2014], Gabor features [Li and Wang, 2013] and other cues [Zhang et al., 2014]. However, the changes of appearance lead to instability of these features. The basic idea of metric learning is to find a mapping function from feature space to distance space with certain merits [Cheng et al., 2016], such as Mahalanobis metric learning (KISSME) [Davis et al., 2007], Information-theoretic metric learning (ITML) [Davis et al., 2007], and large margin nearest neighbour (LMNN) [Weinberger and Saul, 2009]. Both lines of the methods regard feature extraction and metric learning processes as two separate steps, which limit the performances significantly.

Inspired by the great success of deep learning networks in computer vision and pattern recognition tasks [Yang et al., 2017b; Deng et al., 2018; Yang et al., 2018; Li et al., 2018; Yang et al., 2017a; Liu et al., 2016; Yang et al., 2017c], many researchers consider the feature and metric learning jointly in an integrated deep architecture, where feature representation can be learned under supervision of the distance metric loss. In [Li et al., 2014], deep learning is exploited to automatically learn features for the re-identification task. And the deep framework has been improved in [Ahmed et al., 2015] by incorporating neighbouring locations of other images. In [Ding et al., 2015], the relative distance between two images are captured by a triplet loss. To further constrain the distances of pairs, [Cheng et al., 2016] propose an enhance triplet loss function. A multi-task deep network (MTDnet) [Chen et al., 2017] are presented to consider the classification loss and the ranking loss simultaneously and takes advantages both of them during training. In order to jointly extract single-image and cross-image feature representations, [Wang et al., 2016] propose a unified triplet and siamese deep architecture.

\section{Our Architecture}

Fig. 3 shows the framework of the proposed method. The input images of the network are first embedded by the convolution neural network(CNN). Then the output feature maps are respectively projected to different feature subspaces through two full connected layers that do not share parameters. Finally, multiple metric loss functions are used to guide network optimization.

\subsection{View-Aware Feature Embedding}

Given a training set $\left\{\mathbf{x}_{i}, \mathbf{y}_{i}^{1}, \mathbf{y}_{i}^{2}\right\}_{i=1,2, \ldots, n}$, where $\mathbf{x}_{i}$ denotes the $i$ th pedestrian image, $\mathbf{y}_{i}^{1}$ and $\mathbf{y}_{i}^{2}$ are the identity label and the view label of $\mathbf{x}_{i}$ respectively. The view label $\mathbf{y}_{i}^{2}$ is set as 0 when the pedestrian is in front or back views, while as 1 when the pedestrian is in side views. Furthermore, we denote the feature maps of input images extracted by $\mathrm{CNN}$ as $\mathbf{F}=$ $\left\{f\left(\mathbf{x}_{i}\right)\right\}_{i=1,2, \ldots, n}$ and combine them into four kinds of pairs as shown on Figure 2:

$$
\begin{aligned}
& \mathbf{P S}=\left\{\left(f\left(\mathbf{x}_{i}\right), f\left(\mathbf{x}_{j}\right)\right) \mid \mathbf{y}_{i}^{1}=\mathbf{y}_{j}^{1}, \mathbf{y}_{i}^{2}=\mathbf{y}_{j}^{2}\right\} \\
& \mathbf{P D}=\left\{\left(f\left(\mathbf{x}_{i}\right), f\left(\mathbf{x}_{j}\right)\right) \mid \mathbf{y}_{i}^{1}=\mathbf{y}_{j}^{1}, \mathbf{y}_{i}^{2} \neq \mathbf{y}_{j}^{2}\right\} \\
& \mathbf{N S}=\left\{\left(f\left(\mathbf{x}_{i}\right), f\left(\mathbf{x}_{j}\right)\right) \mid \mathbf{y}_{i}^{1} \neq \mathbf{y}_{j}^{1}, \mathbf{y}_{i}^{2}=\mathbf{y}_{j}^{2}\right\} \\
& \mathbf{N D}=\left\{\left(f\left(\mathbf{x}_{i}\right), f\left(\mathbf{x}_{j}\right)\right) \mid \mathbf{y}_{i}^{1} \neq \mathbf{y}_{j}^{1}, \mathbf{y}_{i}^{2} \neq \mathbf{y}_{j}^{2}\right\}
\end{aligned}
$$

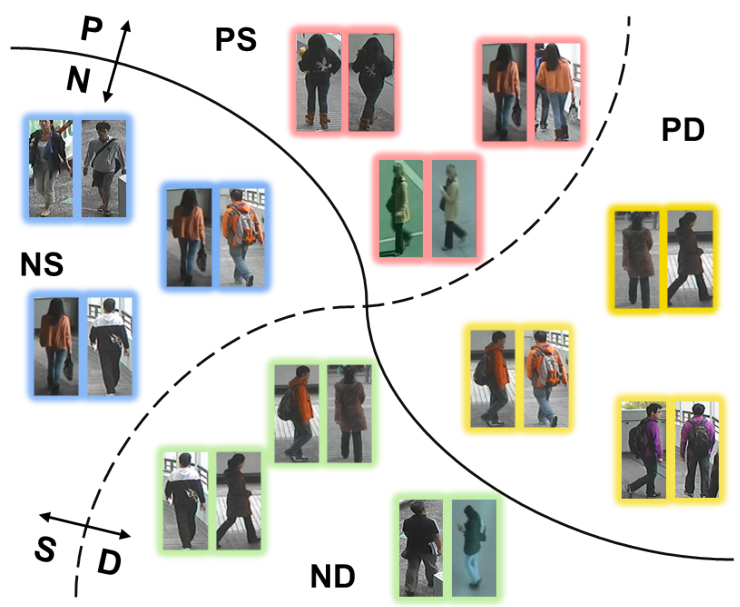

Figure 2: Four sets of image pairs. The active line is the split line between image pairs of same person and of different person, while the broken line is the split line between image pairs in similar views and dissimilar views.

where $1 \leq i, j \leq n$, the feature maps of image pairs $\left(f\left(\mathbf{x}_{i}\right), f\left(\overline{\mathbf{x}}_{j}\right)\right) \in\{\mathbf{P S}, \mathbf{N S}\}$ are projected to one feature subspace by the full connection layer $\mathrm{FC} 1$, while the $\left(f\left(\mathbf{x}_{k}\right), f\left(\mathbf{x}_{l}\right)\right) \in\{\mathbf{P D}, \mathbf{N D}\}$ are projected to another one by FC2. The outputs of FC1 and FC2 are defined as follows:

$$
\begin{aligned}
& g_{1}\left(f\left(\mathbf{x}_{i}\right)\right)=\mathbf{W}_{1} * f\left(\mathbf{x}_{i}\right)+\mathbf{b}_{1} \\
& g_{2}\left(f\left(\mathbf{x}_{k}\right)\right)=\mathbf{W}_{2} * f\left(\mathbf{x}_{k}\right)+\mathbf{b}_{2}
\end{aligned}
$$

where $\mathbf{W}_{1}$ and $\mathbf{W}_{2}$ are the weights of $\mathrm{FC} 1$ and $\mathrm{FC} 2$ while $b_{1}$ and $b_{2}$ represent the biases. $*$ refers to the convolution operation.

The CNN is built inspired by [Xiao et al., 2016; Simonyan and Zisserman, 2014] and details of the structure are listed in Table 1. Each ReLU layer is followed by a Batch Normalization (BN) layer which accelerates the convergence process and avoids manually tweaking the initialization of weights and biases. For training the CNN from scratch, we randomly dropout 50\% neurons of the fc7_1 and fc7_2 layers.

Besides, We learn a view classifier to choose the corresponding feature subspace which the feature maps of images are projected into. The view classifier is optimized by minimizing the sigmoid loss. The first and second layers of the view classifier are convolutional layers with the kernel size $32 \times 7 \times 7$ and $32 \times 5 \times 5$ respectively. The kernels size of third convolutional layer is $64 \times 3 \times 3$ and all the three layers are followed by a pooling layer. Then two fully connected layers that contain 512 neurons receive the output of the third convolutional layer.

\subsection{Multiple Metric Loss}

Different with other methods, we utilize two metric loss functions to constrain the distances between image pairs in similar views and in dissimilar views separately. Figure 4 illustrates the failure case of other methods that constrain the distances between all image pairs with one loss. In Figure 4(a), the loss decreases the distances between $\left(\mathbf{x}_{1}, \mathbf{x}_{2}\right)$ and between 


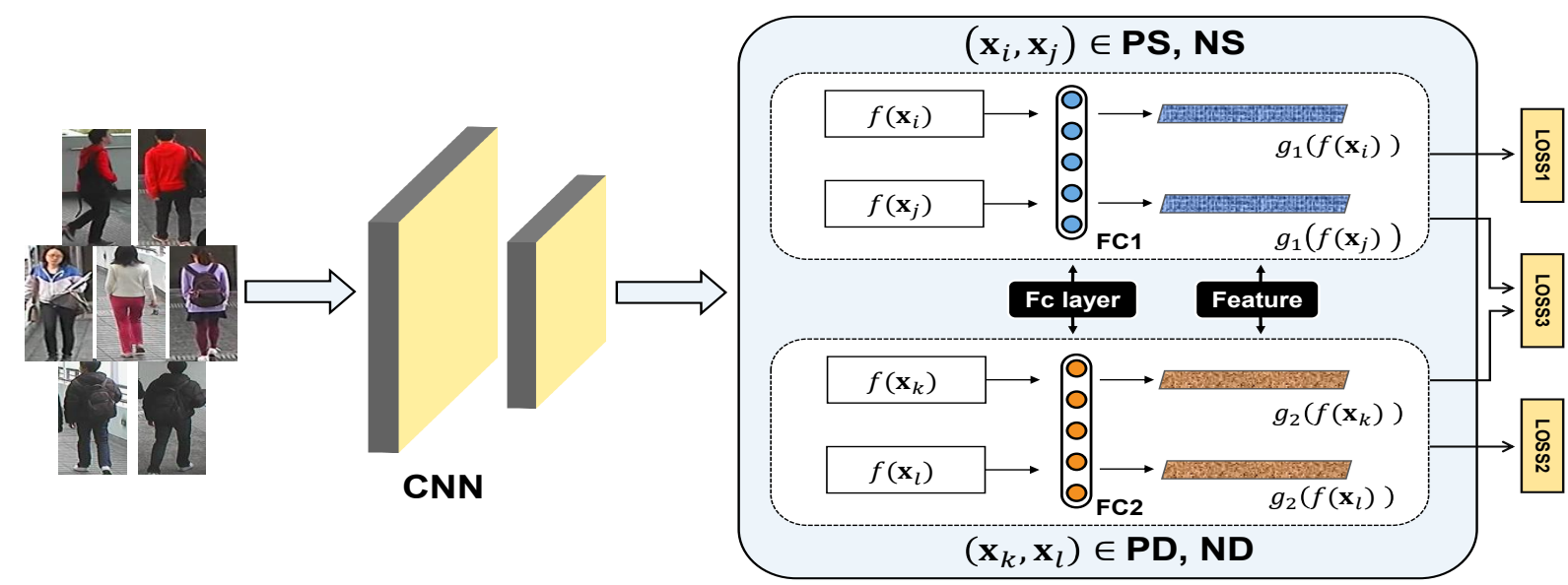

Figure 3: Architecture of the proposed deep view-aware metric learning (DVAML) model. The input of the network is a set of images. Then the feature maps output by the CNN are respectively projected to different feature subspaces through two full connected layers. Finally, three loss functions are used to guide network optimization.

$\left(\mathbf{x}_{1}, \mathbf{x}_{3}\right)$ by giving $\mathbf{x}_{1}$ a upwards and a downwards pulling force respectively. Simultaneously, $\mathbf{x}_{1}$ is given a left and a right pushing force to increase the distances between $\left(\mathbf{x}_{1}, \mathbf{x}_{4}\right)$ and between $\left(\mathbf{x}_{1}, \mathbf{x}_{5}\right)$. Therefore, $\mathbf{x}_{1}$ will still in place. In Figure 4(b), image pairs in similar views and in dissimilar views are first projected into two different feature subspaces as introduced in 3.1 section. Then $L^{1}$ and $L^{2}$ are used to supervise the learning of the different feature subspaces respectively. $L^{1}$ decreases the distance between $\left(\mathbf{x}_{1}, \mathbf{x}_{2}\right)$ by pulling $\mathbf{x}_{1}$ upwards and increases the distance between $\left(\mathbf{x}_{1}, \mathbf{x}_{5}\right)$ by pushing $\mathbf{x}_{1}$ right, while $L^{2}$ decreases the distance between $\left(\mathbf{x}_{1}, \mathbf{x}_{3}\right)$ by pulling $\mathbf{x}_{1}$ downwards and increases the distance between $\left(\mathbf{x}_{1}, \mathbf{x}_{4}\right)$ by pushing $\mathbf{x}_{1}$ left. $\mathbf{x}_{1}$ moves finally towards the correct direction in both feature subspaces.

The loss function $L^{1}$ based on the lifted structured feature embedding[Song et al., 2016] is defined as:

$$
\begin{aligned}
L_{i, j}^{1} & =\log \left[\sum_{(i, k) \in \mathbf{N S}} \exp \left\{\beta_{1}-D_{i, k}\right\}\right. \\
& \left.+\sum_{(j, l) \in \mathbf{N S}} \exp \left\{\beta_{1}-D_{j, l}\right\}\right]+D_{i, j} \\
L^{1} & =\frac{1}{2|\mathbf{P S}|} \sum_{(i, j) \in \mathbf{P S}} \max \left(0, L_{i, j}^{1}\right)^{2}
\end{aligned}
$$

where $L_{i, j}^{1}$ is used to limit the distance between $\mathbf{x}_{i}$ and $\mathbf{x}_{j}$ in PS less than all pairs in NS which contain $\mathbf{x}_{i}$ or $\mathbf{x}_{j}$ with a $\operatorname{margin} \beta_{1}$.

Correspondingly, the loss function $L^{2}$ is defined as:

$$
\begin{aligned}
L_{i, j}^{2} & =\log \left[\sum_{(i, k) \in \mathbf{N D}} \exp \left\{\beta_{2}-D_{i, k}\right\}\right. \\
& \left.+\sum_{(j, l) \in \mathbf{N D}} \exp \left\{\beta_{2}-D_{j, l}\right\}\right]+D_{i, j} \\
L^{2} & =\frac{1}{2|\mathbf{P D}|} \sum_{(i, j) \in \mathbf{P D}} \max \left(0, L_{i, j}^{2}\right)^{2}
\end{aligned}
$$

where $L_{i, j}^{2}$ is used to limit the distance between $\mathbf{x}_{i}$ and $\mathbf{x}_{j}$ in PD less than all pairs in ND which contain $\mathbf{x}_{i}$ or $\mathbf{x}_{j}$ with a margin $\beta_{2}$. The value $|\mathbf{P S}|$ and $|\mathbf{P D}|$ are respectively equal to the number of image pairs belong to PS and PD in each batch.

Noted that the distances between images pairs in similar and dissimilar views are respectively denoted as:

$$
D_{i, j}= \begin{cases}\left\|g_{1}\left(f\left(\mathbf{x}_{i}\right)\right)-g_{1}\left(f\left(\mathbf{x}_{j}\right)\right)\right\|_{2} & (i, j) \in \mathbf{S} \\ \left\|g_{2}\left(f\left(\mathbf{x}_{i}\right)\right)-g_{2}\left(f\left(\mathbf{x}_{j}\right)\right)\right\|_{2} & (i, j) \in \mathbf{D}\end{cases}
$$

in which $\mathbf{S}$ denotes the set of image pairs in similar views including PS and NS, while $\mathbf{D}$ denotes the set of image pairs with dissimilar views consisting of $\mathbf{P D}$ and ND.

Considering that the loss function $L^{1}$ and $L^{2}$ only constrains the distances between image pairs in similar views and dissimilar views separately. So we use $L^{3}$ constrain the distances between image pairs of same person and between image pairs of different person overall. The loss function $L^{3}$ is defined as:

$$
\begin{aligned}
L_{i, j}^{3} & =\log \left[\sum_{(i, k) \in \mathbf{N}} \exp \left\{\beta_{3}-D_{i, k}\right\}\right. \\
& \left.+\sum_{(j, l) \in \mathbf{N}} \exp \left\{\beta_{3}-D_{j, l}\right\}\right]+D_{i, j} \\
L^{3}= & \frac{1}{2|\mathbf{P S}|+|\mathbf{P D}|} \sum_{(i, j) \in \mathbf{P}} \max \left(0, L_{i, j}^{3}\right)^{2}
\end{aligned}
$$

in which $\mathbf{P}$ denotes the set of the image pairs of same identity consist of PS and PD. $\mathbf{N}$ constrains all the image pairs in NS and ND.

We finally unite the three loss functions to a joint loss function:

$$
L=\alpha_{1} L^{1}+\alpha_{2} L^{2}+\alpha_{3} L^{3}
$$

where the three hyper-parameters $\alpha_{1}, \alpha_{2}$ and $\alpha_{3}$ are used to balance the three loss functions. 


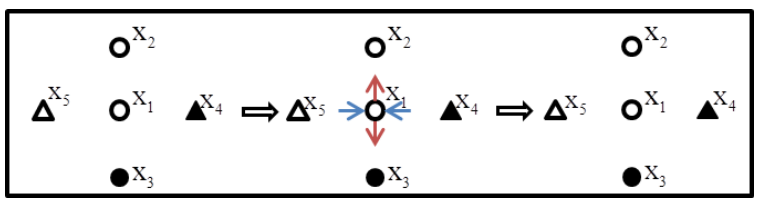

(a)

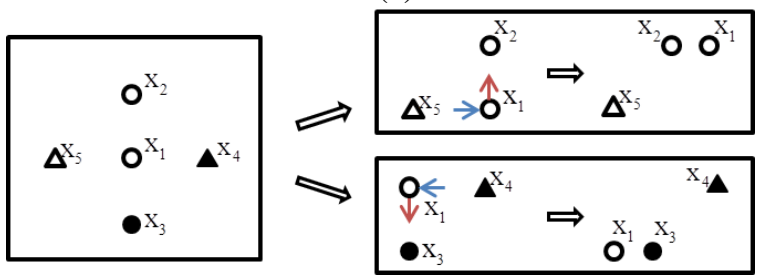

(b)

Figure 4: The rounds $\mathbf{x}_{1}, \mathbf{x}_{2}$ and $\mathbf{x}_{3}$ are images of person $\mathrm{A}$, while the triangles $\mathbf{x}_{4}$ and $\mathbf{x}_{5}$ are images of person $\mathrm{B}$. The solid and hollow denote the two different views of the person respectively. The red arrowhead means the pulling force and the blue arrowhead means the push force.

\subsection{Optimization}

The network is jointly supervised and optimized by the joint loss. The back propagation gradient with respect to the distance $D_{i, j}$ is:

$$
\begin{aligned}
\frac{\partial L}{\partial D_{i, j}} & =\frac{\partial L^{1}}{\partial D_{i, j}} \mathbf{1}[(i, j) \in \mathbf{P S}] \\
& +\frac{\partial L^{2}}{\partial D_{i, j}} \mathbf{1}[(i, j) \in \mathbf{P D}]+\frac{\partial L^{3}}{\partial D_{i, j}}
\end{aligned}
$$

where 1 is the indicator function which outputs 1 if the expression evaluates to true and outputs 0 otherwise. The gradients of the distances between image pairs in PS consist of two components coming from $L^{1}$ and $L^{3}$, while that of image pairs in PD coming from $L^{2}$ and $L^{3}$.

$$
\begin{gathered}
\frac{\partial L^{1}}{\partial D_{i, j}}=\frac{1}{|\mathbf{P S}|} L_{i, j}^{1} \mathbf{1}\left[L_{i, j}^{1}>0\right] \\
\frac{\partial L^{2}}{\partial D_{i, j}}=\frac{1}{|\mathbf{P D}|} L_{i, j}^{2} \mathbf{1}\left[L_{i, j}^{2}>0\right] \\
\frac{\partial L^{3}}{\partial D_{i, j}}=\frac{1}{|\mathbf{P S}|+|\mathbf{P D}|} L_{i, j}^{3} \mathbf{1}\left[L_{i, j}^{3}>0\right]
\end{gathered}
$$

The gradient with respect to the distance $D_{i, k}$ is:

$$
\begin{aligned}
\frac{\partial L}{\partial D_{i, k}} & =\frac{\partial L^{1}}{\partial D_{i, k}} \mathbf{1}[(i, k) \in \mathbf{N S}] \\
& +\frac{\partial L^{2}}{\partial D_{i, k}} \mathbf{1}[(i, k) \in \mathbf{N D}]+\frac{\partial L^{3}}{\partial D_{i, k}}
\end{aligned}
$$

in which the gradients of the three loss functions with respect to $D_{i, k}$ are:

$$
\frac{\partial L^{1}}{\partial D_{i, k}}=\frac{L_{i, j}^{1} \mathbf{1}\left[L_{i, j}^{1}>0\right]}{|\mathbf{P S}|} \sum_{(i, j) \in \mathbf{P S}} \frac{-\exp \left\{\beta_{1}-D_{i, k}\right\}}{\exp \left\{L_{i, j}^{1}-D_{i, j}\right\}}
$$

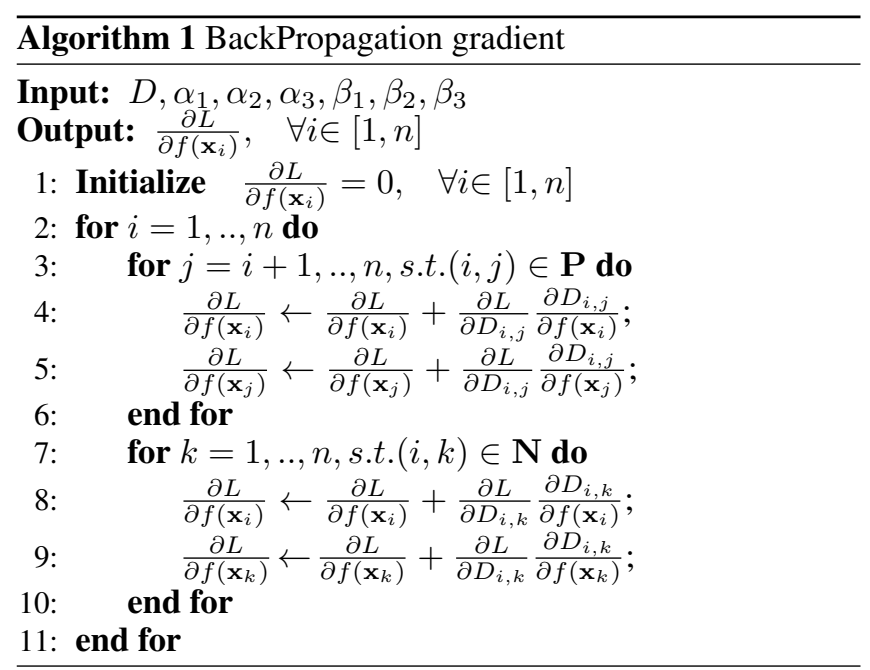

$\frac{\partial L^{2}}{\partial D_{i, k}}=\frac{L_{i, j}^{2} \mathbf{1}\left[L_{i, j}^{2}>0\right]}{|\mathbf{P D}|} \sum_{(i, j) \in \mathbf{P D}} \frac{-\exp \left\{\beta_{2}-D_{i, k}\right\}}{\exp \left\{L_{i, j}^{2}-D_{i, j}\right\}}$

$$
\frac{\partial L^{3}}{\partial D_{i, k}}=\frac{L_{i, j}^{3} \mathbf{1}\left[L_{i, j}^{3}>0\right]}{|\mathbf{P S}|+|\mathbf{P D}|} \sum_{(i, j) \in \mathbf{P}} \frac{-\exp \left\{\beta_{3}-D_{i, k}\right\}}{\exp \left\{L_{i, j}^{3}-D_{i, j}\right\}}
$$

The gradient with respect to $D_{j, l}$ is:

$$
\begin{aligned}
\frac{\partial L}{\partial D_{j, l}} & =\frac{\partial L^{1}}{\partial D_{j, l}} \mathbf{1}[(j, l) \in \mathbf{N S}] \\
& +\frac{\partial L^{2}}{\partial D_{j, l}} \mathbf{1}[(j, l) \in \mathbf{N D}]+\frac{\partial L^{3}}{\partial D_{j, l}}
\end{aligned}
$$

The gradients of the distances between image pairs in similar views with respect to the feature maps of them are:

$$
\begin{aligned}
\frac{\partial D_{i, j}}{\partial f\left(\mathbf{x}_{i}\right)} & =\frac{1}{D_{i, j}}\left(g_{1}\left(f\left(\mathbf{x}_{i}\right)\right)-g_{1}\left(f\left(\mathbf{x}_{j}\right)\right)\right) \frac{\partial g_{1}\left(f\left(\mathbf{x}_{i}\right)\right)}{\partial f\left(\mathbf{x}_{i}\right)} \\
\frac{\partial D_{i, j}}{\partial f\left(\mathbf{x}_{j}\right)} & =\frac{1}{D_{i, j}}\left(g_{1}\left(f\left(\mathbf{x}_{j}\right)\right)-g_{1}\left(f\left(\mathbf{x}_{i}\right)\right)\right) \frac{\partial g_{1}\left(f\left(\mathbf{x}_{j}\right)\right)}{\partial f\left(\mathbf{x}_{j}\right)}
\end{aligned}
$$

Correspondingly, the gradients of the distances between image pairs in dissimilar views with respect to the feature maps of them are:

$$
\begin{gathered}
\frac{\partial D_{i, j}}{\partial f\left(\mathbf{x}_{i}\right)}=\frac{1}{D_{i, j}}\left(g_{2}\left(f\left(\mathbf{x}_{i}\right)\right)-g_{2}\left(f\left(\mathbf{x}_{j}\right)\right)\right) \frac{\partial g_{2}\left(f\left(\mathbf{x}_{i}\right)\right)}{\partial f\left(\mathbf{x}_{i}\right)} \\
\frac{\partial D_{i, j}}{\partial f\left(\mathbf{x}_{j}\right)}=\frac{1}{D_{i, j}}\left(g_{2}\left(f\left(\mathbf{x}_{j}\right)\right)-g_{2}\left(f\left(\mathbf{x}_{i}\right)\right)\right) \frac{\partial g_{2}\left(f\left(\mathbf{x}_{j}\right)\right)}{\partial f\left(\mathbf{x}_{j}\right)}
\end{gathered}
$$

\section{Experiments}

\subsection{Datasets and Settings}

Datasets: We evaluate our method on three datasets, CUHK03 [Li et al., 2014], CUHK01 [Li et al., 2012] and PRID2011 [Hirzer et al., 2011]. CUHK03 dataset contains 13164 images from 1360 persons. We select 1160 persons 
Proceedings of the Twenty-Seventh International Joint Conference on Artificial Intelligence (IJCAI-18)

\begin{tabular}{|c|c|c|c|c|c|c|c|c|}
\hline Type & $\begin{array}{c}\text { patch size/ } \\
\text { stride }\end{array}$ & $\begin{array}{c}\text { output } \\
\text { size }\end{array}$ & $\# 1 \times 1$ & $\begin{array}{l}\# 3 \times 3 \\
\text { reduce } \\
\end{array}$ & $\# 1 \times 1$ & $\begin{array}{c}\text { double } \# 3 \times 3 \\
\text { reduce }\end{array}$ & $\begin{array}{c}\text { double } \\
\# 3 \times 3 \\
\end{array}$ & pool + proj \\
\hline input & & $3 \times 128 \times 64$ & & & & & & \\
\hline conv1-conv2 & $3 \times 3 / 2$ & $32 \times 128 \times 64$ & & & & & & \\
\hline conv3 & $3 \times 3 / 2$ & $64 \times 128 \times 64$ & & & & & & \\
\hline pool3 & $2 \times 2 / 2$ & $64 \times 64 \times 32$ & & & & & & \\
\hline inception (4a) & & $256 \times 64 \times 32$ & 64 & 64 & 64 & 64 & 64 & avg +64 \\
\hline inception (4b) & & $384 \times 32 \times 16$ & 64 & 64 & 64 & 64 & 64 & max + pass through \\
\hline inception (5a) & & $512 \times 32 \times 16$ & 128 & 128 & 128 & 128 & 128 & avg +128 \\
\hline inception (5b) & & $768 \times 16 \times 8$ & 128 & 128 & 128 & 128 & 128 & max + pass through \\
\hline inception (6a) & & $1024 \times 16 \times 8$ & 256 & 256 & 256 & 256 & 256 & avg +256 \\
\hline inception (6b) & & $1536 \times 8 \times 4$ & 256 & 256 & 256 & 256 & 256 & max + pass through \\
\hline avg pool & $8 \times 4 / 1$ & $1536 \times 1 \times 1$ & & & & & & \\
\hline fc7_1 & & $512 \times 1 \times 1$ & & & & & & \\
\hline fc7_2 & & $512 \times 1 \times 1$ & & & & & & \\
\hline
\end{tabular}

Table 1: The network details of CNN in the proposed method for person re-identification

\begin{tabular}{|l|c|c|c|c|}
\hline Method & Top1 & Top5 & Top10 & Top20 \\
\hline \hline KISSME & 14.17 & 37.46 & 52.20 & 69.32 \\
\hline ITML & 5.53 & 18.89 & 28.96 & 44.33 \\
\hline LMNN & 21.17 & 49.49 & 61.12 & 78.32 \\
\hline eSDC & 8.76 & 24.07 & 39.28 & 50.91 \\
\hline FPNN & 20.65 & 51.00 & 67.00 & 83.31 \\
\hline kLFDA & 48.20 & 69.01 & 79.63 & 89.18 \\
\hline IDLA & 54.74 & 86.50 & 94.00 & 98.00 \\
\hline GatedSiamese & 68.10 & 88.10 & 94.60 & - \\
\hline MTDnet & 74.68 & 95.99 & 97.47 & - \\
\hline Ours & $\mathbf{7 6 . 1 1}$ & $\mathbf{9 6 . 0 1}$ & $\mathbf{9 8 . 9 0}$ & $\mathbf{9 9 . 8 7}$ \\
\hline
\end{tabular}

Table 2: Matching rates (\%) on CUHK03 dataset

for training, 100 for validation and 100 for testing following the same setting as [Li et al., 2014] and [Ahmed et al., 2015]. CUHK01 [Li et al., 2012] dataset contains 971 persons captured from two camera views in a campus. We utilize the same protocol used in [Zhou et al., 2017] and [Wang et al., 2016], where 871 person images are used for training and the left for testing. Following the setting in [Chen et al., 2017], 100 persons in PRID2011 dataset are used for training, specially, 100 for probe and 649 (the remaining persons from camera B except the 100 for training) for gallery in test set.

For training the view classifier, we manually annotate the coarse view labels for 200 persons in CUHK03 and CUHK01. Considering the size and complexity of PRID2011 dataset, 50 persons in it are annotated. Since the view classification is treated as a simple binary classification problem, the view classifier achieves a high accuracy rate even though training samples are limited.

Considering that multiple cameras in varied views may be retrieved for a given query image in practical application, we fuse the three datasets two by two into three new datasets: Syn1 (CUHK03 and CUHK01), Syn2 (CUHK03 and PRID2011) and Syn3 (CUHK01 and PRID2011). Then we train the model and retrieve probe of the one on the two datasets for each fused. For example, the same 1260 person$\mathrm{s}$ in CUHK03 and 871 persons in CUHK01 are chosen as Syn1's training set. For the probe of CUHK03, there are an-

\begin{tabular}{|l|c|c|c|c|}
\hline Method & Top1 & Top5 & Top10 & Top20 \\
\hline \hline KISSME & 29.40 & 59.34 & 71.45 & 88.12 \\
\hline ITML & 17.10 & 42.31 & 55.07 & 71.65 \\
\hline LMNN & 21.17 & 49.49 & 61.12 & 78.32 \\
\hline eSDC & 22.84 & 43.89 & 57.67 & 69.84 \\
\hline FPNN & 27.87 & 58.20 & 73.46 & 86.31 \\
\hline kLFDA & 42.76 & 69.01 & 79.63 & 89.18 \\
\hline IDLA & 65.00 & 89.33 & 93.00 & 96.51 \\
\hline ImpTrpLoss & 53.70 & 84.30 & 91.00 & 96.30 \\
\hline P2S & 77.34 & 93.51 & 96.73 & 98.53 \\
\hline MTDnet-cross & 78.50 & $\mathbf{9 6 . 5 0}$ & 97.50 & - \\
\hline Ours & $\mathbf{8 0 . 5 4}$ & 95.00 & $\mathbf{9 7 . 9 9}$ & $\mathbf{9 9 . 3 8}$ \\
\hline
\end{tabular}

Table 3: Matching rates (\%) on CUHK01 dataset

\begin{tabular}{|l|c|c|c|c|}
\hline Method & Top1 & Top5 & Top10 & Top20 \\
\hline \hline KISSME & 28.54 & 59.78 & 72.13 & 83.26 \\
\hline LMNN & 14.38 & 38.09 & 50.22 & 67.19 \\
\hline kLFDA & 22.10 & 46.60 & 58.10 & - \\
\hline LADF & 8.20 & 20.45 & 29.89 & 42.25 \\
\hline ImpTrpLoss & 22.00 & - & 47.00 & 57.00 \\
\hline MDTnet-cross & 31.00 & 54.00 & 61.00 & - \\
\hline Ours & $\mathbf{3 3 . 0 0}$ & $\mathbf{6 5 . 0 0}$ & $\mathbf{7 5 . 0 0}$ & $\mathbf{8 3 . 0 0}$ \\
\hline
\end{tabular}

Table 4: Matching rates (\%) on PRID-2011 dataset

other 100 persons from CUHK01 in the CUHK03's gallery besides the original 100. Equally, there are 200 persons in the CUHK01's gallery. Noted that 749 persons are used as the gallery of PRID2011 in Syn2 and Syn3, while only 200 persons as the CUHK01's and CUHK03's gallery.

Parameters Implementation. All the images are resized to $128 * 64$ before being fed to the network and the batchsize of the input is 64 . For identity classifier, we first pretrain a model on CUHK03 and the learning rate is set to 0.001 , then finetune this model with learning rate 0.002 . We picked a set of optimal loss weights $\alpha_{1}=0.4, \alpha_{2}=0.4, \alpha_{3}=0.2$ experimentally. And all the margin parameters $\beta_{1}, \beta_{2}, \beta_{3}$ are set to 1 [Song et al., 2016].

Evaluation Protocol: We report the single-shot results on 
Proceedings of the Twenty-Seventh International Joint Conference on Artificial Intelligence (IJCAI-18)

\begin{tabular}{|l|c|c|c|c|c|c|}
\hline \multirow{2}{*}{ Method } & \multicolumn{3}{|c|}{ CUHK03 } & \multicolumn{3}{c|}{ CUHK01 } \\
\cline { 2 - 7 } & Top1 & Top5 & Top10 & Top1 & Top5 & Top10 \\
\hline \hline KISSME & 11.03 & 32.21 & 49.82 & 21.32 & 52.17 & 68.44 \\
\hline LMNN & 18.33 & 46.31 & 57.52 & 17.52 & 45.32 & 56.31 \\
\hline kLFDA & 43.17 & 67.32 & 72.63 & 37.65 & 65.32 & 74.33 \\
\hline MTDnet & 67.00 & 68.00 & 92.00 & 70.00 & 91.00 & 96.00 \\
\hline Ours & $\mathbf{7 2 . 7 7}$ & $\mathbf{9 4 . 8 3}$ & $\mathbf{9 8 . 0 0}$ & $\mathbf{7 3 . 0 0}$ & $\mathbf{9 3 . 0 0}$ & $\mathbf{9 7 . 0 0}$ \\
\hline
\end{tabular}

Table 5: Matching rates (\%) on Syn1 dataset

the three datasets and the synthetic dataset of them. The datasets are separated into the training set and the testing set, and the testing set is further divided into probe set and gallery set that contain different images of the same person. The result is evaluated by cumulative matching characteristic (CMC) curve, which is an estimation of finding the corrected match in the top $n$ match.

\subsection{Results and Analysis}

We compare our method with 7 common methods including KISSME, ITML, LMNN, eSDC [Zhao et al., 2013b], kLFDA [Xiong et al., 2014], FPNN [Li et al., 2014], IDLA [Ahmed et al., 2015]. And Table 2-7 show the evaluation results of these method.

Results on CUHK03. In Table 2, we also compare our method with two deep methods GatedSiamese [Varior et al., 2016] and MTDnet [Chen et al., 2017] except the 7 methods. From Table1, most of deep learning methods except FPNN obviously outperform the traditional methods. Ours model has achieved the top performances by $76.11 \%$ on Top 1 accuracy (vs. $74.68 \%$ by the next best method).

Results on CUHK01. Table 3 extra lists the results of the P2S [Zhou et al., 2017] and ImpTrpLoss [Cheng et al., 2016] methods. From Table 3, we can see that our method outperforms the state-of-the-art methods by more than $80 \%$ on Top1 accuracy ( $80.54 \%$ vs. $78.50 \%$ by MTDnet-cross). Note that the Top 5 recognition rate of our method reaches $95.00 \%$, meaning that the trained model has high probability of finding the correct person from other cameras.

Results on PRID2011. We can see from Table 4 that our method achieves $33 \%$ on Top 1 matching rate and outperforms the previous best accuracy on PRID2011 even though the training samples are limited for deep neural networks. It is noted that the Top 5 matching rate achieves $65 \%$ far beyond the accuracy of other methods.

Results on Syn1, Syn2 and Syn3. Table 5-7 illustrates the performance of ours method and the previous methods on synthetic datasets. And it's obvious that our method has achieved the top performance on all the three datasets. From Table 5, we can see that the matching rate of our method on CUHK03 and CUHK01 reaches $72.77 \%$ and $73.00 \%$. It's worth noting that the accuracy on Syn1 declines contrast to each single dataset due to the gallery images from the other dataset can interfere the retrieval of the probe. The results on Syn 2 are listed in Table 6 and our method's accuracy achieves $75.02 \%$ and $35.00 \%$ on CUHK03 and PRID2011 respectively. The accuracy of PRID2011 when trained on Syn2 dataset improves $2 \%$ compared with on single dataset, since

\begin{tabular}{|l|c|c|c|c|c|c|}
\hline \multirow{2}{*}{ Method } & \multicolumn{3}{|c|}{ CUHK03 } & \multicolumn{3}{c|}{ PRID2011 } \\
\cline { 2 - 7 } & Top1 & Top5 & Top10 & Top1 & Top5 & Top10 \\
\hline \hline KISSME & 13.56 & 32.66 & 48.63 & 24.31 & 48.41 & 68.13 \\
\hline LMNN & 19.54 & 47.26 & 60.21 & 12.98 & 35.67 & 46.85 \\
\hline kLFDA & 45.31 & 65.33 & 77.39 & 19.69 & 43.74 & 56.31 \\
\hline MTDnet & 72.00 & 91.00 & 94.00 & 21.00 & 41.00 & 52.00 \\
\hline Ours & $\mathbf{7 5 . 0 2}$ & $\mathbf{9 4 . 5 1}$ & $\mathbf{9 6 . 3 6}$ & $\mathbf{3 5 . 0 0}$ & $\mathbf{6 1 . 0 0}$ & $\mathbf{7 0 . 0 0}$ \\
\hline
\end{tabular}

Table 6: Matching rates (\%) on Syn2 dataset

\begin{tabular}{|l|c|c|c|c|c|c|}
\hline \multirow{2}{*}{ Method } & \multicolumn{3}{|c|}{ CUHK01 } & \multicolumn{3}{c|}{ PRID2011 } \\
\cline { 2 - 7 } & Top1 & Top5 & Top10 & Top1 & Top5 & Top10 \\
\hline \hline KISSME & 14.57 & 35.61 & 52.63 & 26.31 & 51.41 & 65.13 \\
\hline LMNN & 20.61 & 45.26 & 62.33 & 14.92 & 37.62 & 44.80 \\
\hline kLFDA & 47.21 & 68.35 & 74.32 & 18.61 & 45.72 & 55.31 \\
\hline MTDnet & 73.00 & 91.00 & 93.00 & 24.00 & 39.00 & 47.00 \\
\hline Ours & $\mathbf{7 6 . 0 0}$ & $\mathbf{9 1 . 2 7}$ & $\mathbf{9 4 . 0 0}$ & $\mathbf{3 1 . 0 0}$ & $\mathbf{6 5 . 0 0}$ & $\mathbf{7 7 . 0 0}$ \\
\hline
\end{tabular}

Table 7: Matching rates (\%) on Syn3 dataset

the added training samples may improve the performance of model on small dataset. The accuracy of CUHK01 and PRID2011 listed in Table 7 reaches $76.00 \%$ and $31.00 \%$. It is noted that we improve the performance on PRID2011 by more than $20 \%$ on the rank-5 accuracy in contrast to the previous methods.

Effect of deep view-aware metric. We employ an viewaware method to project image pairs into different feature subspaces. The method has positive effect on person reidentification since it captures the intrinsic relevance between image pairs. Besides, the proposed network is constrained by three metric loss functions, of which the two supervise separately the learning of the different feature subspaces and the last supervises the whole network. And our model wins over $3 \%$ compared to other methods on the three synthetic dataset$\mathrm{s}$, which further proves the effectiveness of it.

\section{Conclusion}

In this paper, we propose a deep view-aware metric learning (DVAML) model for person re-identification. We project image pairs in similar and dissimilar views into different feature subspaces, respectively. Then multiple loss functions are used to supervise the learning of the different feature subspaces. In addition, we also consider the actual situation that a given probe image can be retrieved from multiple cameras by synthesizing different datasets. Experiment results on CUHK03, CUHK01, PRID2011 and three synthetic datasets illustrate that our method outperforms the state-of-the-art approaches.

\section{Acknowledgments}

This work was supported in part by the National Natural Science Foundation of China under Grant 61572388 and Grant 61703327, and in part by the Key R\&D Program. The Key Industry Innovation Chain of Shaanxi under Grant 2017ZDCXL-GY-05-04-02 and Grant 2017ZDCXL-GY-0504-02. 


\section{References}

[Ahmed et al., 2015] Ejaz Ahmed, Michael Jones, and Tim K. Marks. An improved deep learning architecture for person re-identification. In CVPR, 2015.

[Chen et al., 2017] Weihua Chen, Xiaotang Chen, Jianguo Zhang, and Kaiqi Huang. A multi-task deep network for person re-identification. In $A A A I, 2017$.

[Cheng et al., 2016] De Cheng, Yihong Gong, Sanping Zhou, Jinjun Wang, and Nanning Zheng. Person reidentification by multi-channel parts-based cnn with improved triplet loss function. In CVPR, 2016.

[Davis et al., 2007] Jason V. Davis, Brian Kulis, Prateek Jain, Suvrit Sra, and Inderjit S. Dhillon. Informationtheoretic metric learning. In ICML, 2007.

[Deng et al., 2018] Cheng Deng, Zhaojia Chen, Xianglong Liu, Xinbo Gao, and Dacheng Tao. Triplet-based deep hashing network for cross-modal retrieval. IEEE Transactions on Image Processing, 2018.

[Ding et al., 2015] Shengyong Ding, Liang Lin, Guangrun Wang, and Hongyang Chao. Deep feature learning with relative distance comparison for person re-identification. Pattern Recognition, 2015.

[Hirzer et al., 2011] Martin Hirzer, Csaba Beleznai, Peter M Roth, and Horst Bischof. Person re-identification by descriptive and discriminative classification. In Scandinavian conference on Image analysis. Springer, 2011.

[Khamis et al., 2014] Sameh Khamis, Cheng-Hao Kuo, Vivek K Singh, Vinay D Shet, and Larry S Davis. Join$\mathrm{t}$ learning for attribute-consistent person re-identification. In $E C C V, 2014$.

[Li and Wang, 2013] Wei Li and Xiaogang Wang. Locally aligned feature transforms across views. In CVPR, 2013.

[Li et al., 2012] Wei Li, Rui Zhao, and Xiaogang Wang. Human reidentification with transferred metric learning. In $A C C V, 2012$.

[Li et al., 2014] Wei Li, Rui Zhao, Tong Xiao, and Xiaogang Wang. Deepreid: Deep filter pairing neural network for person re-identification. In $C V P R, 2014$.

[Li et al., 2018] Chao Li, Cheng Deng, Ning Li, Wei Liu, Xinbo Gao, and Dacheng Tao. Self-supervised adversarial hashing networks for cross-modal retrieval. arXiv preprint arXiv:1804.01223, 2018.

[Liu et al., 2016] Xianglong Liu, Cheng Deng, Bo Lang, Dacheng Tao, and Xuelong Li. Query-adaptive reciprocal hash tables for nearest neighbor search. IEEE Transactions on Image Processing, 2016.

[Simonyan and Zisserman, 2014] Karen Simonyan and Andrew Zisserman. Very deep convolutional networks for large-scale image recognition. arXiv preprint arXiv:1409.1556, 2014.

[Song et al., 2016] Hyun Oh Song, Yu Xiang, Stefanie Jegel$\mathrm{ka}$, and Silvio Savarese. Deep metric learning via lifted structured feature embedding. In $C V P R, 2016$.
[Varior et al., 2016] Rahul Rama Varior, Mrinal Haloi, and Gang Wang. Gated siamese convolutional neural network architecture for human re-identification. In ECCV, 2016.

[Wang et al., 2016] Faqiang Wang, Wangmeng Zuo, Liang Lin, David Zhang, and Lei Zhang. Joint learning of single-image and cross-image representations for person re-identification. In $C V P R, 2016$.

[Weinberger and Saul, 2009] Kilian Q Weinberger and Lawrence K Saul. Distance metric learning for large margin nearest neighbor classification. Journal of Machine Learning Research, 2009.

[Xiao et al., 2016] Tong Xiao, Hongsheng Li, Wanli Ouyang, and Xiaogang Wang. Learning deep feature representations with domain guided dropout for person re-identification. In $C V P R, 2016$.

[Xiong et al., 2014] Fei Xiong, Mengran Gou, Octavia Camps, and Mario Sznaier. Person re-identification using kernel-based metric learning methods. In ECCV, 2014.

[Yang et al., 2017a] Erkun Yang, Cheng Deng, Wei Liu, Xianglong Liu, Dacheng Tao, and Xinbo Gao. Pairwise relationship guided deep hashing for cross-modal retrieval. In AAAI, 2017.

[Yang et al., 2017b] Yanhua Yang, Cheng Deng, Shangqian Gao, Wei Liu, Dapeng Tao, and Xinbo Gao. Discriminative multi-instance multitask learning for $3 \mathrm{~d}$ action recognition. IEEE Transactions on Multimedia, 2017.

[Yang et al., 2017c] Yanhua Yang, Cheng Deng, Dapeng Tao, Shaoting Zhang, Wei Liu, and Xinbo Gao. Latent max-margin multitask learning with skelets for 3-d action recognition. IEEE Transactions on Cybernetics, 2017.

[Yang et al., 2018] Erkun Yang, Cheng Deng, Chao Li, Wei Liu, Jie Li, and Dacheng Tao. Shared predictive crossmodal deep quantization. IEEE TNNLS, 2018.

[Zhang et al., 2014] Ziming Zhang, Yuting Chen, and Venkatesh Saligrama. A novel visual word co-occurrence model for person re-identification. In ECCV, 2014.

[Zhang et al., 2016] Yaqing Zhang, Xi Li, Liming Zhao, and Zhongfei Zhang. Semantics-aware deep correspondence structure learning for robust person re-identification. In IJCAI, 2016.

[Zhao et al., 2013a] Rui Zhao, Wanli Ouyang, and Xiaogang Wang. Person re-identification by salience matching. In ICCV, 2013.

[Zhao et al., 2013b] Rui Zhao, Wanli Ouyang, and Xiaogang Wang. Unsupervised salience learning for person reidentification. In CVPR, 2013.

[Zhao et al., 2017] Haiyu Zhao, Maoqing Tian, Shuyang Sun, Jing Shao, Junjie Yan, Shuai Yi, Xiaogang Wang, and Xiaoou Tang. Spindle net person re-identification with human body region guided feature decomposition and fusion. In CVPR, 2017.

[Zhou et al., 2017] Sanping Zhou, Jinjun Wang, Jiayun Wang, Yihong Gong, and Nanning Zheng. Point to set similarity based deep feature learning for person reidentification. In $C V P R, 2017$. 